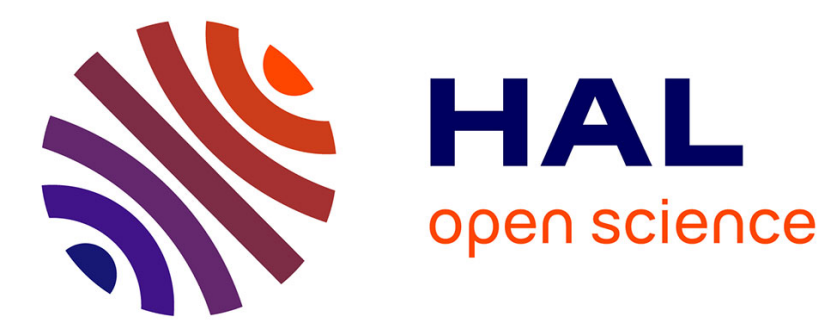

\title{
On the high-SNR conditional maximum-likelihood estimator full statistical characterization
}

Alexandre Renaux, Philippe Forster, Eric Chaumette, Pascal Larzabal

\section{To cite this version:}

Alexandre Renaux, Philippe Forster, Eric Chaumette, Pascal Larzabal. On the high-SNR conditional maximum-likelihood estimator full statistical characterization. IEEE Transactions on Signal Processing, 2006, 54 (12), pp.4840-4843. inria-00444708

\section{HAL Id: inria-00444708 \\ https://hal.inria.fr/inria-00444708}

Submitted on 8 Jan 2010

HAL is a multi-disciplinary open access archive for the deposit and dissemination of scientific research documents, whether they are published or not. The documents may come from teaching and research institutions in France or abroad, or from public or private research centers.
L'archive ouverte pluridisciplinaire HAL, est destinée au dépôt et à la diffusion de documents scientifiques de niveau recherche, publiés ou non, émanant des établissements d'enseignement et de recherche français ou étrangers, des laboratoires publics ou privés. 


\title{
On the High SNR Conditional
}

\section{Maximum-Likelihood Estimator Full Statistical Characterization}

\author{
Alexandre Renaux, Philippe Forster, Member, IEEE, Eric Chaumette, and Pascal Larzabal, Member, \\ IEEE
}

\begin{abstract}
In the field of asymptotic performance characterization of Conditional Maximum Likelihood (CML) estimator, asymptotic generally refers to either the number of samples or the Signal to Noise Ratio (SNR) value. The first case has been already fully characterized although the second case has been only partially investigated. Therefore, this correspondence aims to provide a sound proof of a result, i.e. asymptotic (in SNR) Gaussianity and efficiency of the CML estimator in the multiple parameters case, generally regarded as trivial but not so far demonstrated.
\end{abstract}

\section{Index Terms}

Maximum Likelihood, statistical efficiency, high Signal to Noise Ratio, array processing.

\section{INTRODUCTION}

Parameters estimation of multiple signals impinging on an antenna array is a fundamental problem in signal processing with applications to radar, sonar, digital communication and many other fields. A plethora of algorithms have been proposed in the literature in this sense (see [1]).

Perhaps the most well known and frequently used model-based approach in signal processing is the Maximum Likelihood (ML) technique. When applying the ML technique to a sensors array problem, two main methods have been considered, depending on the model used for the signal waveforms. When the source signals are modeled as Gaussian random processes, a Stochastic ML (SML) is obtained. If, on the other hand, when the source signals are modeled as unknown deterministic quantities, the resulting estimator is referred to as the CML estimator (see, e.g.[2], for a review of the two methods).

A. Renaux and P. Larzabal are with Ecole Normale Superieure of Cachan, SATIE Laboratory, 61, avenue du President Wilson 94235 Cachan, France. (e-mail: renaux@satie.ens-cachan.fr).

P. Forster is with University Paris 10, GEA laboratory, 1 Chemin Desvallieres, 92410 Ville d'Avray, France.

E. Chaumette is with Thales Naval France, 6-9 avenue des mathurins, 92200 Bagneux, France.

This worh has been performed in the framework of the European Community Contract no. 507325, NEWCOM 
Asymptotic statistical performance of these ML methods is an important field of research. For that purpose, the estimation accuracy is generally investigated by means of the Cramér-Rao bound. Since two models are used for the different ML methods, two Cramér-Rao bounds have been derived: the stochastic Cramér-Rao bound when the source signals are modelled as Gaussian random processes and the deterministic Cramér-Rao bound when the source signals are modelled as unknown deterministic quantities (see, e.g.[2], for a review of these two bounds).

In the array processing context, the term "asymptotic" can be understood in two different ways: in the number of samples or in the SNR value. At large number of samples, the statistical performance of these ML methods has been fully characterized (see [3]). Concerning the high SNR context, the non-efficiency (in comparison with the stochastic Cramér-Rao bound) and the non-Gaussianity of the SML have been recently proved in [4]. Concerning the CML method in the high SNR framework, it is generally accepted that this estimator is Gaussian and efficient although, to our knowledge, there is no sound proof of this result in the literature in the multi-parameters case. Indeed, to the best of our knowledge, the CML estimator has been only partially investigated in [5] where the Gaussianity of the CML estimates is proved in the single parameter case by the way of a Gaussian observation model with parameterized mean. Moreover, the asymptotic efficiency of the CML estimator in the high SNR case has never been demonstrated. This correspondence aims to complete Kay's result, i.e. to establish the Gaussianity and the efficiency (in comparison with the deterministic Cramér-Rao bound) of the CML estimator in the multiple parameters case. Moreover, we show how these results still hold for non-circular complex Gaussian noise. Monte Carlo simulations are provided in order to show the accuracy of the analysis.

The notational convention adopted is as follows: italic indicates a scalar quantity, as in $A$; lower case boldface indicates a vector quantity, as in $\mathbf{a}$; upper case boldface indicates a matrix quantity, as in $\mathbf{A}$. The $n$-th row and $m$-th column element of the matrix $\mathbf{A}$ will be denoted by $A_{n, m} . \operatorname{Re}\{A\}$ is the real part of $A$ and $\operatorname{Im}\{A\}$ is the imaginary part of $A$. The matrix transpose is indicated by a superscript ${ }^{\mathrm{T}}$ as in $\mathbf{A}^{\mathrm{T}}$. $|\mathbf{A}|$ is the determinant of the square matrix A. $\mathbf{I}_{M}$ is the identity matrix of order $M . E[\cdot]$ denotes the expectation operator and $\|$.$\| the norm.$ A sample of a random vector $\mathbf{a}$ is denoted $\mathbf{a}(\omega)$ where $\omega$ belongs to the event space $\Omega . o($.$) and o_{p}($.$) denote$ respectively the small oh and the stochastic small oh notation.

\section{OBSERVATION MODEL AND MAXIMUM LIKELIHOOD ESTIMATOR}

\section{A. Observation Model}

In the sequel, we consider the following general observation model

$$
\mathbf{x}(\omega)=\mathbf{m}\left(\mathbf{p}_{0}\right)+\mathbf{n}(\omega),
$$

where $\mathbf{x}(\omega)$ is a real $M \times 1$ sample vector, $\omega \in \Omega, \mathbf{p}=\left[p_{1}, \ldots, p_{P}\right]^{\mathrm{T}}$ is the $P \times 1(P \leq M)$ real vector of unknown deterministic parameters of interest with true value $\mathbf{p}_{0}, \mathbf{m}$ is a $M \times 1$ real deterministic vector depending (generally non-linearly) on $\mathbf{p}$ which is assumed to be identifiable from $\mathbf{m}(\mathbf{p})$. $\mathbf{n}(\omega)$ is the $M \times 1$ additive noise vector which is a sample of a random Gaussian, zero mean vector $\mathbf{n}$ with covariance matrix $\sigma^{2} \mathbf{C}$. $\mathbf{C}$ is assumed to be known 
and $\sigma^{2}$ is unknown ${ }^{1}$.

The $\log$-likelihood $\ln f\left(\mathbf{x}(\omega) ; \mathbf{p}, \sigma^{2}\right)$ of the observations is

$$
\begin{aligned}
\ln f\left(\mathbf{x}(\omega) ; \mathbf{p}, \sigma^{2}\right) & =-\frac{1}{2} \ln \left((2 \pi)^{M}\left|\sigma^{2} \mathbf{C}\right|\right) \\
& -\frac{1}{2 \sigma^{2}}(\mathbf{x}(\omega)-\mathbf{m}(\mathbf{p}))^{\mathrm{T}} \mathbf{C}^{-1}(\mathbf{x}(\omega)-\mathbf{m}(\mathbf{p})) .
\end{aligned}
$$

Let us note that the study with the observation $\breve{\mathbf{x}}(\omega)=\breve{\mathbf{m}}\left(\mathbf{p}_{0}\right)+\breve{\mathbf{n}}(\omega)$ where $\breve{\mathbf{x}}(\omega), \breve{\mathbf{m}}$ and $\breve{\mathbf{n}}(\omega)$ are complex can be handled by the real model (1). Indeed, by stacking the real and imaginary parts of $\breve{\mathbf{x}}(\omega), \breve{\mathbf{m}}$ and $\breve{\mathbf{n}}(\omega)$, one obtains

$$
\left(\begin{array}{c}
\operatorname{Re}\{\breve{\mathbf{x}}(\omega)\} \\
\operatorname{Im}\{\breve{\mathbf{x}}(\omega)\}
\end{array}\right)=\left(\begin{array}{c}
\operatorname{Re}\left\{\breve{\mathbf{m}}\left(\mathbf{p}_{0}\right)\right\} \\
\operatorname{Im}\left\{\breve{\mathbf{m}}\left(\mathbf{p}_{0}\right)\right\}
\end{array}\right)+\left(\begin{array}{c}
\operatorname{Re}\{\breve{\mathbf{n}}(\omega)\} \\
\operatorname{Im}\{\breve{\mathbf{n}}(\omega)\}
\end{array}\right),
$$

which is similar to the Eqn. (1). Modifications of Eqn. (2) are straightforward and lead to an augmented covariance matrix $(2 M \times 2 M)$ instead of $\mathbf{C}$ taking into account the possible non-circularity of the noise [6].

So, the two following important problems in the array processing context can be statistically characterized in the framework of model (1):

- The CML method (with the notations of Eqn. (4.16) of [2]):

$$
\mathbf{X}_{N}=\mathbf{A}\left(\boldsymbol{\theta}_{0}\right) \mathbf{S}_{N}+\mathbf{N}_{N}
$$

by setting

$$
\mathbf{m}(\mathbf{p})=\left[\operatorname{Re}\left(\operatorname{vec}\left(\mathbf{A}\left(\boldsymbol{\theta}_{0}\right) \mathbf{S}_{N}\right)\right)^{\mathrm{T}} \quad \operatorname{Im}\left(\operatorname{vec}\left(\mathbf{A}\left(\boldsymbol{\theta}_{0}\right) \mathbf{S}_{N}\right)\right)^{\mathrm{T}}\right]^{\mathrm{T}},
$$

where $\mathbf{p}=\left[\boldsymbol{\theta}^{\mathrm{T}}, \operatorname{vec}\left(\mathbf{S}_{N}\right)^{\mathrm{T}}\right]^{\mathrm{T}}$.

- The so-called known waveforms model (see e.g. [7]) (with the notations of Eqn. (8) of [7])

$$
\mathbf{x}(t)=\mathbf{A}\left(\boldsymbol{\theta}_{0}\right) \mathbf{P}(t) \boldsymbol{\alpha}+\mathbf{n}(t)
$$

by setting

$$
\mathbf{m}(\mathbf{p})=\left[\begin{array}{ll}
\operatorname{Re}\left(v e c\left(\mathbf{A}\left(\boldsymbol{\theta}_{0}\right) \mathbf{P}(t) \boldsymbol{\alpha}\right)\right)^{\mathrm{T}} & \operatorname{Im}\left(\operatorname{vec}\left(\mathbf{A}\left(\boldsymbol{\theta}_{0}\right) \mathbf{P}(t) \boldsymbol{\alpha}\right)\right)^{\mathrm{T}}
\end{array}\right]^{\mathrm{T}},
$$

where $\mathbf{p}=\left[\boldsymbol{\theta}^{\mathrm{T}}, \boldsymbol{\alpha}^{\mathrm{T}}\right]^{\mathrm{T}}$. This model finds applications in active Radar and in data-aided processing for mobile communications.

\section{B. Maximum Likelihood estimator}

The ML estimate of $\mathbf{p}$ is given by

$$
\hat{\mathbf{p}}(\omega)=\arg \min _{\mathbf{p}}\left\{(\mathbf{x}(\omega)-\mathbf{m}(\mathbf{p}))^{\mathrm{T}} \mathbf{C}^{-1}(\mathbf{x}(\omega)-\mathbf{m}(\mathbf{p}))\right\} .
$$

The solution of (8) is obtained by setting the gradient of the criterion $(\mathbf{x}(\omega)-\mathbf{m}(\mathbf{p}))^{\mathrm{T}} \mathbf{C}^{-1}(\mathbf{x}(\omega)-\mathbf{m}(\mathbf{p}))$ equal to zero. This leads to the set of $P$ equations where the dependence on $\mathbf{n}(\omega)$ is explicitly shown

$$
\left.\mathbf{g}(\mathbf{p}, \mathbf{n}(\omega))\right|_{\mathbf{p}=\hat{\mathbf{p}}(\omega)}=\mathbf{0},
$$

\footnotetext{
${ }^{1}$ The high SNR analysis is equivalent to an analysis of $\sigma \rightarrow 0$.
} 
where the elements of $\mathbf{g}=\left[g_{1}(\mathbf{p}, \mathbf{n}(\omega)), \cdots, g_{P}(\mathbf{p}, \mathbf{n}(\omega))\right]^{T}$, for $i=1, \ldots, P$, are given by

$$
\begin{aligned}
g_{i}(\mathbf{p}, \mathbf{n}(\omega)) & =(\mathbf{x}(\omega)-\mathbf{m}(\mathbf{p}))^{\mathrm{T}} \mathbf{C}^{-1} \frac{\partial \mathbf{m}(\mathbf{p})}{\partial p_{i}} \\
& =\left(\mathbf{m}\left(\mathbf{p}_{0}\right)-\mathbf{m}(\mathbf{p})+\mathbf{n}(\omega)\right)^{\mathrm{T}} \mathbf{C}^{-1} \frac{\partial \mathbf{m}(\mathbf{p})}{\partial p_{i}} .
\end{aligned}
$$

\section{PERFORMANCE AT HIGH SNR}

An improvement on the well known Kay approach [5] is to resort to the implicit function theorem (see [8] theorem 9.28): indeed, this theorem not only provides a framework for a sound demonstration of the asymptotical Gaussian behavior of CML estimator, but also, the analytical expression of the asymptotic estimator covariance matrix that allows to establish the asymptotic efficiency.

\section{A. Background: the implicit function theorem}

Let $\mathbf{f}(\mathbf{u}, \mathbf{v})=\left[f_{1}(\mathbf{u}, \mathbf{v}), \cdots, f_{P}(\mathbf{u}, \mathbf{v})\right]^{T}$ be a function of $I R^{P} \times I R^{M} \rightarrow I R^{P}$. Let us assume that

- Assumption Al: $f_{i}(\mathbf{u}, \mathbf{v})$ for $i=1, \ldots, P$ are differentiable functions on a neighborhood of the point $\left(\mathbf{u}_{0}, \mathbf{v}_{0}\right)$ in $I R^{P} \times I R^{M}$.

- Assumption A2: $f_{i}\left(\mathbf{u}_{0}, \mathbf{v}_{0}\right)=0$ for $i=1, \ldots, P$.

- Assumption A3: the $P \times P$ Jacobian matrix $\boldsymbol{\Phi}$ of $f_{i}(\mathbf{u}, \mathbf{v})$ with respect to $\mathbf{u}$ is nonsingular at $\left(\mathbf{u}_{0}, \mathbf{v}_{0}\right)$.

Then there is a neighborhood $V$ of the point $\mathbf{v}_{0}$ in $I R^{M}$, there is a neighborhood $U$ of the point $\mathbf{u}_{0}$ in $I R^{P}$, and there is a unique mapping $\varphi: V \rightarrow U$ such that $\varphi\left(\mathbf{v}_{0}\right)=\mathbf{u}_{0}$ and $f_{i}(\boldsymbol{\varphi}(\mathbf{v}), \mathbf{v})=0$ for $i=1, \ldots, P$ and for all $\mathbf{v}$ in $V$. Furthermore, $\varphi$ is differentiable and we have

$$
\boldsymbol{\varphi}(\mathbf{v})-\mathbf{u}_{0}=-\boldsymbol{\Phi}^{-1} \boldsymbol{\Psi}\left(\mathbf{v}-\mathbf{v}_{0}\right)+\mathbf{r}\left(\mathbf{v}-\mathbf{v}_{0}\right)
$$

where the remainder $\mathbf{r}\left(\mathbf{v}-\mathbf{v}_{0}\right)=o\left(\left\|\mathbf{v}-\mathbf{v}_{0}\right\|\right)$ and, by setting $\mathbf{u}=\left[u_{1}, \ldots, u_{P}\right]^{\mathrm{T}}$ and $\mathbf{v}=\left[v_{1}, \ldots, v_{M}\right]^{\mathrm{T}}$,

$$
\boldsymbol{\Phi}=\left[\left.\frac{\partial \mathbf{f}}{\partial u_{1}}\right|_{\left(\mathbf{u}_{0}, \mathbf{v}_{0}\right)}, \cdots,\left.\frac{\partial \mathbf{f}}{\partial u_{P}}\right|_{\left(\mathbf{u}_{0}, \mathbf{v}_{0}\right)}\right],
$$

and

$$
\boldsymbol{\Psi}=\left[\left.\frac{\partial \mathbf{f}}{\partial v_{1}}\right|_{\left(\mathbf{u}_{0}, \mathbf{v}_{0}\right)}, \cdots,\left.\frac{\partial \mathbf{f}}{\partial v_{M}}\right|_{\left(\mathbf{u}_{0}, \mathbf{v}_{0}\right)}\right] .
$$

\section{B. Application to the criterion gradient}

Let us now detail the structure of $\boldsymbol{\Phi}$ and $\boldsymbol{\Psi}$ for the specific function $g$ of Eqn. (10). For the elements of $\Psi$, we have

$$
\Psi_{i, j}=\left.\frac{\partial g_{i}(\mathbf{u}, \mathbf{v})}{\partial v_{j}}\right|_{\left(\mathbf{u}_{0}, \mathbf{v}_{0}\right)}=\left.\frac{\partial \mathbf{v}^{\mathrm{T}}}{\partial v_{j}} \mathbf{C}^{-1} \frac{\partial \mathbf{m}}{\partial u_{i}}\right|_{\left(\mathbf{u}_{0}, \mathbf{v}_{0}\right)},
$$

with $1 \leq i \leq P$ and $1 \leq j \leq M$. Concerning the elements of $\boldsymbol{\Phi}$, we have

$$
\Phi_{i, j}=\left.\frac{\partial g_{i}(\mathbf{u}, \mathbf{v})}{\partial u_{j}}\right|_{\left(\mathbf{u}_{0}, \mathbf{v}_{0}\right)}=-\left.\frac{\partial \mathbf{m}^{\mathrm{T}}}{\partial u_{j}} \mathbf{C}^{-1} \frac{\partial \mathbf{m}}{\partial u_{i}}\right|_{\mathbf{u}_{0}}
$$

with $1 \leq i, j \leq P$. 
In other words,

$$
\boldsymbol{\Psi}=\frac{\partial \mathbf{m}^{\mathrm{T}}}{\partial \mathbf{u}} \mathbf{C}^{-1} \frac{\partial \mathbf{v}}{\partial \mathbf{v}^{\mathrm{T}}} \text { and } \boldsymbol{\Phi}=-\frac{\partial \mathbf{m}^{\mathrm{T}}}{\partial \mathbf{u}} \mathbf{C}^{-1} \frac{\partial \mathbf{m}}{\partial \mathbf{u}^{\mathrm{T}}} .
$$

One can notice that $\boldsymbol{\Phi}$ is linked to $\boldsymbol{\Psi}$ by

$$
\boldsymbol{\Phi}=-\boldsymbol{\Psi} \mathbf{C} \boldsymbol{\Psi}^{\mathrm{T}}
$$

since $\frac{\partial \mathbf{v}}{\partial \mathbf{v}^{T}}=\mathbf{I}_{M}$.

Let us now apply the implicit function theorem by setting $\mathbf{v}=\mathbf{n}(\omega), \boldsymbol{\varphi}(\mathbf{v})=\hat{\mathbf{p}}(\omega), \mathbf{u}_{0}=\mathbf{p}_{0}$ and $\mathbf{v}_{0}=\mathbf{0}$. Let us note that $A 2$ is obviously satisfied since, without noise, the criterion $(\mathbf{x}(\omega)-\mathbf{m}(\mathbf{p}))^{\mathrm{T}} \mathbf{C}^{-1}(\mathbf{x}(\omega)-\mathbf{m}(\mathbf{p}))$ is minimum and equal to $\mathbf{0}$ for $\mathbf{p}=\mathbf{p}_{0} . A 1$ and $A 3$ will be assumed to be satisfied in the sequel.

Consequently, the implicit function theorem leads to

$$
\hat{\mathbf{p}}(\omega)-\mathbf{p}_{\mathbf{0}}=-\boldsymbol{\Phi}^{-1} \mathbf{\Psi} \mathbf{n}(\omega)+\mathbf{r}(\mathbf{n}(\omega)) \quad \forall \omega \in \Omega
$$

\section{Asymptotic Gaussianity of the ML estimator}

Let us set $\mathbf{n}_{k}$ a sequence of a Gaussian random noise with zero means and covariance matrices $\sigma_{k}^{2} \mathbf{C}$. $\sigma_{k}$ is a sequence such as $\sigma_{k} \rightarrow 0$ when $k \rightarrow \infty$. Let us set $\hat{\mathbf{p}}_{k}(\omega)$ and $\mathbf{n}_{k}(\omega)$ two sequences of the random vector $\hat{\mathbf{p}}_{k}$ and $\mathbf{n}_{k}$ respectively. $\hat{\mathbf{p}}_{k}(\omega)$ is the corresponding solution of (9). Let us set $\Delta \mathbf{p}_{k}(\omega)=\frac{1}{\sigma_{k}}\left(\hat{\mathbf{p}}_{k}(\omega)-\mathbf{p}_{0}\right)$ a sequence of the random vector $\Delta \mathbf{p}_{k}$. Then, the high SNR analysis of the ML estimator is given by the behavior of the random vector $\Delta \mathbf{p}_{k}$ when $k \rightarrow \infty$. From Eqn. (18) we have

$$
\Delta \mathbf{p}_{k}(\omega)=-\boldsymbol{\Phi}^{-1} \boldsymbol{\Psi} \frac{\mathbf{n}_{k}(\omega)}{\sigma_{k}}+\frac{\mathbf{r}\left(\mathbf{n}_{k}(\omega)\right)}{\sigma_{k}} \quad \forall \omega \in \Omega,
$$

or equivalently in terms of random vectors $\mathbf{n}_{k}$ and $\Delta \mathbf{p}_{k}$

$$
\Delta \mathbf{p}_{k}=-\boldsymbol{\Phi}^{-1} \boldsymbol{\Psi} \frac{\mathbf{n}_{k}}{\sigma_{k}}+\frac{\mathbf{r}\left(\mathbf{n}_{k}\right)}{\sigma_{k}} .
$$

By using lemma 2.12 (i) of [9] we have

$$
\mathbf{r}\left(\mathbf{n}_{k}\right)=o_{p}\left(\left\|\mathbf{n}_{k}\right\|\right)
$$

We will now study the two terms of the right hand side of Eqn. (20). For that purpose, note that $\frac{\mathbf{n}_{k}}{\sigma_{k}}$ follows a Gaussian distribution with zero mean and covariance matrix $\mathbf{C}$. The term $\frac{\mathbf{r}\left(\mathbf{n}_{k}\right)}{\sigma_{k}}=\frac{o_{p}\left(\left\|\mathbf{n}_{k}\right\|\right)}{\sigma_{k}}$ can be rewritten as

$$
\frac{o_{p}\left(\left\|\mathbf{n}_{k}\right\|\right)}{\sigma_{k}}=\frac{\left\|\mathbf{n}_{k}\right\|}{\sigma_{k}} \frac{o_{p}\left(\left\|\mathbf{n}_{k}\right\|\right)}{\left\|\mathbf{n}_{k}\right\|},
$$

where $\frac{o_{p}\left(\left\|\mathbf{n}_{k}\right\|\right)}{\left\|\mathbf{n}_{k}\right\|}$ converges in probability to zero by definition and where $\frac{\left\|\mathbf{n}_{k}\right\|}{\sigma_{k}}$ follows a chi law which does not depend on $k$. Consequently, $\frac{o_{p}\left(\left\|\mathbf{n}_{k}\right\|\right)}{\sigma_{k}}$ converges in probability to zero (see [10] pp.122).

Concerning the term $-\boldsymbol{\Phi}^{-1} \boldsymbol{\Psi} \frac{\mathbf{n}_{k}}{\sigma_{k}}$, it follows a Gaussian distribution with zero mean and covariance matrix $\boldsymbol{\Gamma}$ equal to

$$
\begin{aligned}
\boldsymbol{\Gamma} & =\boldsymbol{\Phi}^{-1} \boldsymbol{\Psi} \mathbf{C}\left(\boldsymbol{\Phi}^{-1} \boldsymbol{\Psi}\right)^{\mathrm{T}} \\
& =\boldsymbol{\Phi}^{-1} \boldsymbol{\Psi} \mathbf{C} \boldsymbol{\Psi}^{\mathrm{T}} \boldsymbol{\Phi}^{-\mathrm{T}} \\
& =-\boldsymbol{\Phi}^{-1}=\left(\boldsymbol{\Psi} \mathbf{C} \boldsymbol{\Psi}^{\mathrm{T}}\right)^{-1}
\end{aligned}
$$


thanks to Eqn. (17). Consequently, $\Delta \mathbf{p}_{k}$ converges in distribution to a zero mean Gaussian random vector with covariance $\boldsymbol{\Gamma}=\left(\mathbf{\Psi} \mathbf{C} \boldsymbol{\Psi}^{\mathrm{T}}\right)^{-1}$ when $k \rightarrow \infty$ or equivalently when $\sigma_{k} \rightarrow 0$. This proves the asymptotic Gaussianity of the ML estimator at high SNR.

\section{Asymptotic efficiency of the ML estimator}

The closed-form of the error covariance matrix obtained by the means of the implicit function theorem allows to establish the asymptotic efficiency of the ML estimator by a direct comparison with the Cramér-Rao Lower Bound (CRLB). For that purpose, let us compute the Fisher Information Matrix (FIM) which reduces to a block diagonal form thanks to the decoupling between parameter $\mathbf{p}$ and $\sigma^{2}[11]$

$$
\boldsymbol{F I M}\left(\mathbf{p}_{0}, \sigma^{2}\right)=\left(\begin{array}{cc}
\mathbf{F I M}\left(\mathbf{p}_{0}\right) & \mathbf{0} \\
\mathbf{0} & \mathbf{F I M}\left(\sigma^{2}\right)
\end{array}\right) .
$$

Consequently, the CRLB for $\mathbf{p}_{0}$ is given by

$$
\begin{aligned}
\operatorname{CRLB}\left(\mathbf{p}_{0}\right) & =\mathbf{F I M}^{-1}\left(\mathbf{p}_{0}\right) \\
& =-\left(E\left[\frac{\partial^{2} \ln f\left(\mathbf{x} ; \mathbf{p}, \sigma^{2}\right)}{\partial \mathbf{p} \partial \mathbf{p}^{T}}\right]\right)^{-1} \\
& =2 \sigma^{2}\left(E\left[\frac{\partial^{2}(\mathbf{x}-\mathbf{m}(\mathbf{p}))^{\mathrm{T}} \mathbf{C}^{-1}(\mathbf{x}-\mathbf{m}(\mathbf{p}))}{\partial \mathbf{p} \partial \mathbf{p}^{T}}\right]\right)^{-1} \\
& =-\sigma^{2}\left(E\left[\frac{\partial \mathbf{g}(\mathbf{p}, \mathbf{n})}{\partial \mathbf{p}^{T}}\right]\right)^{-1} \\
& =-\sigma^{2} \mathbf{\Phi}^{-1}=\sigma^{2}\left(\mathbf{\Psi} \mathbf{C} \Psi^{\mathbf{T}}\right)^{-1}
\end{aligned}
$$

Finally, the ML estimator is efficient at high SNR since

$$
\frac{1}{\sigma^{2}} \operatorname{CRLB}\left(\mathbf{p}_{\mathbf{0}}\right)=\boldsymbol{\Gamma} \text {. }
$$

\section{Simulation RESUlts}

In this section, simulations are performed to illustrate the validity of our analysis in the framework of the CML estimator described in [2]. The CRLB is computed according to Eqn. (4.68) of [2]. In all simulations, the array is a Uniform Linear Array of $M=10$ sensors with half-wavelength spacing. Direction-Of-Arrivals (DOA) are given with respect to the broadside. Monte Carlo simulations have been performed with $r=1000$ independent realizations. The ML DOA estimation is performed with a Gauss Newton algorithm thanks to a global search over a grid.

\section{A. Efficiency}

Let us consider the case of two equi-powered sources located at 0 deg and 3 deg (the array beamwidth is equal to $10 \mathrm{deg}$ ). The CML DOA estimation is performed with $T=10$ snapshots. Figure 1 displays the behavior of the CML empirical variance and the CRLB versus SNR. As expected, the efficiency of the CML estimator at high SNR is observed. 

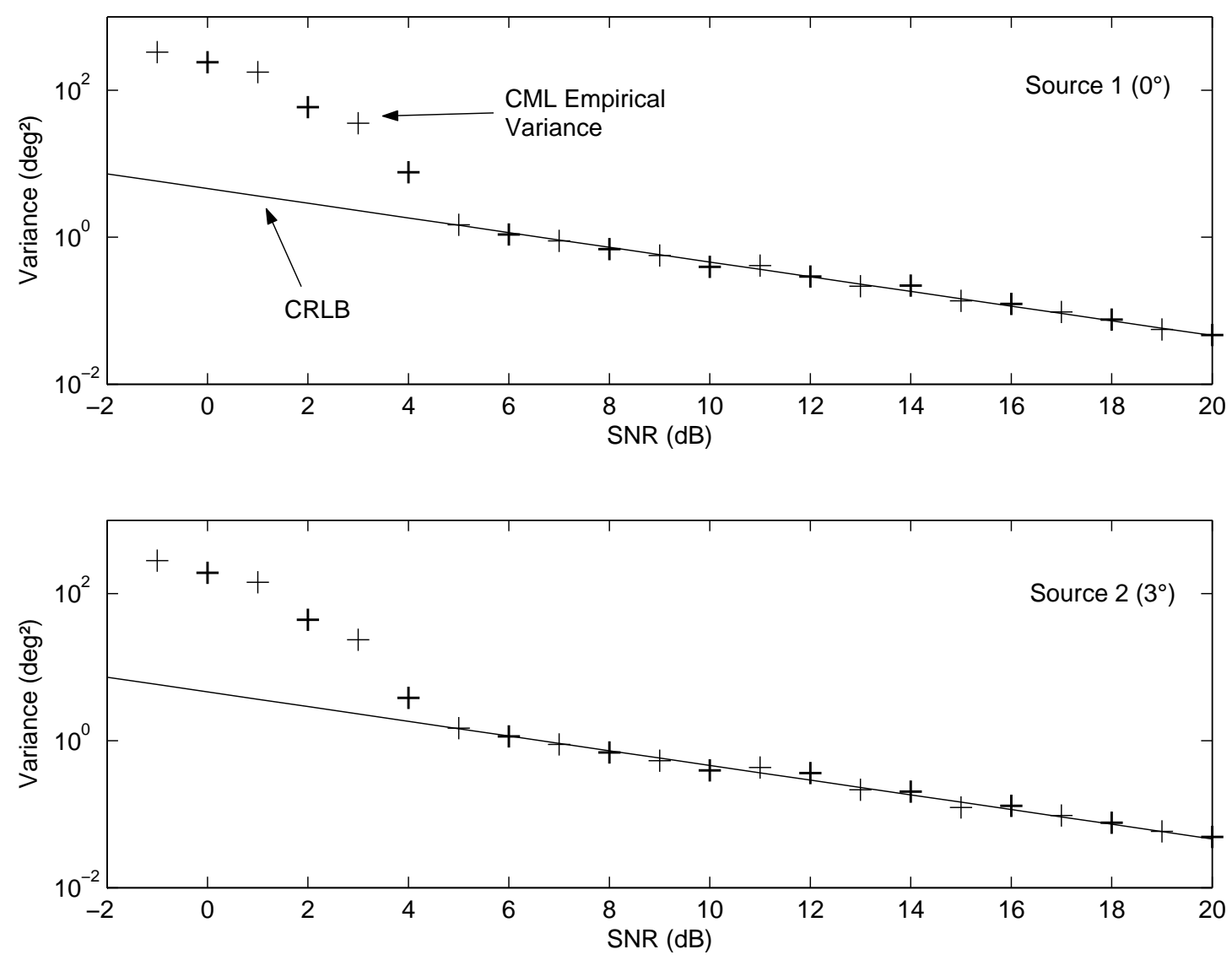

Fig. 1. CRLB and empirical variance of the CML estimator versus SNR for two sources. $M=10$ sensors and $T=10$ snapshots.

We also observe the well known threshold effect [12] of the estimator variance when the SNR becomes weak (approximatively $5 \mathrm{~dB}$ in this case). This phenomena due to outliers gives the validity domain in term of efficiency of this asymptotic analysis (see [13] for more details concerning the CML threshold prediction).

\section{B. Gaussianity}

In order to emphasize the high SNR Gaussianity of the CML estimator, we have used a Lilliefors goodness-of-fit test [14]. This test evaluates the hypothesis that a sample $\left[y_{1} \cdots y_{L}\right]$ has a normal distribution with unspecified mean and variance against the alternative hypothesis that the sample does not have a normal distribution. This test is close to the well known Kolmogorov-Smirnov test which requires the specification of the mean and the variance.

The Lilliefors test statistic $T_{\text {stat }}$ is the maximum vertical distance between the empirical cumulative distribution function $F(s)$ of the score series

$$
z_{k}=\left(y_{k}-\frac{1}{L} \sum_{i=1}^{L} y_{i}\right) / \sqrt{\frac{1}{L} \sum_{j=1}^{L}\left(y_{j}-\frac{1}{L} \sum_{i=1}^{L} y_{i}\right)^{2}}
$$


and the cumulative distribution function of the standard normal distribution $Q(s)$. In other words

$$
T_{\text {stat }}=\sup _{s \in \mathbb{R}}|F(s)-Q(s)| .
$$

The Gaussianity hypothesis is rejected at the significance level $\alpha$ (equal to $5 \%$ in the following exemple) if $T$ exceeds the $1-\alpha$ quantile in a Lilliefors table of quantiles [15] equals to $\frac{0.886}{\sqrt{r}}$ for $r>30$. Here, this cutoff value is $\frac{0.886}{\sqrt{r}}=0.028$.

The test has been performed for the aforementioned scenario (two sources located at 0 deg and 3 deg, 10 snapshots, 10 sensors, 1000 runs). Figure 2 displays the behavior of $T_{\text {stat }}$ versus SNR. The Gaussianity of the CML estimator is observed for an SNR higher than approximatively $9 \mathrm{~dB}$.
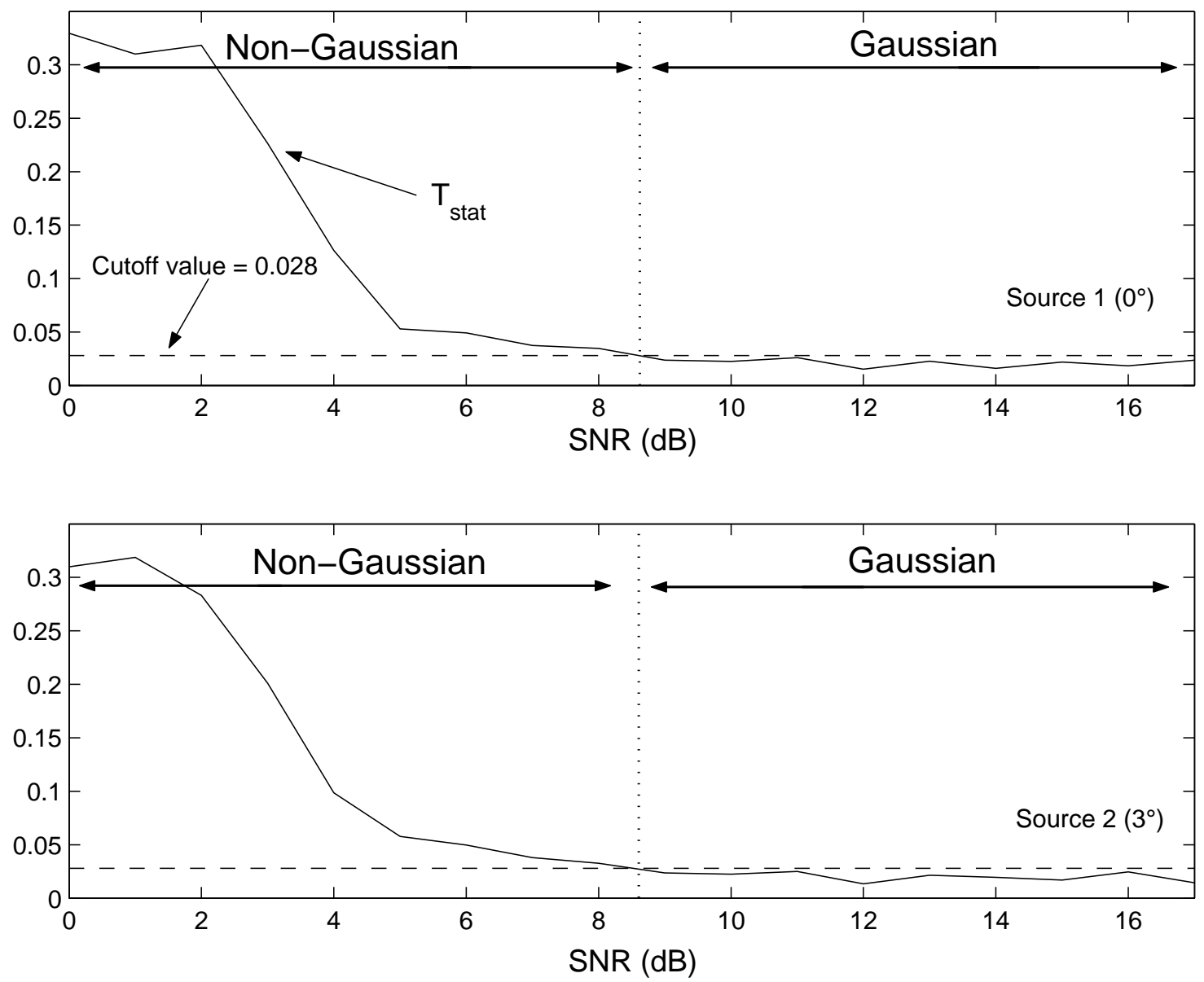

Fig. 2. Lilliefors test $T_{\text {stat }}$ and cutoff value for the CML estimates versus SNR. $M=10$ sensors, $T=10$ snapshots, $r=1000$ runs.

\section{Conclusion}

In this correspondence, we have completed initial Kay works on the CML characterization at high SNR. Thanks to the implicit function theorem, we have provided a sound proof of its asymptotic Gaussianity and efficiency in 
the multiple parameters case.

\section{REFERENCES}

[1] H. Krim and M. Viberg, "Two decades of array signal processing research: the parametric approach," IEEE Signal Processing Mag., vol. 13, no. 4, pp. 67-94, 1996.

[2] B. Ottersten, M. Viberg, P. Stoica, and A. Nehorai, "Exact and large sample maximum likelihood techniques for parameter estimation and detection in array processing," in Radar Array Processing (S. Haykin, J. Litva, and T. J. Shepherd, eds.), ch. 4, pp. 99-151, Berlin: Springer-Verlag, 1993.

[3] P. Stoica and A. Nehorai, "Performances study of conditional and unconditional direction of arrival estimation," IEEE Trans. Acoust., Speech, Signal Processing, vol. 38, pp. 1783-1795, Oct. 1990.

[4] A. Renaux, P. Forster, E. Boyer, and P. Larzabal, "Non-efficiency and non-Gaussianity of a maximum likelihood estimator at high signal to noise ratio and finite number of samples," in Proc. IEEE Int. Conf. Acoust., Speech, Signal Processing, vol. 2, (Montreal, QC), pp. 121-124, May 2004.

[5] S. M. Kay, Fundamentals of Statistical Signal Processing, vol. 1. NJ: Prentice Hall, 1993.

[6] A. V. D. Bos, "The multivariate complex normal distribution - A generalization," IEEE Trans. Inform. Theory, vol. 41, pp. 537-539, Mar. 1995.

[7] J. Li and R. T. Compton, "Maximum likelihood angle estimation for signals with known waveforms," IEEE Trans. Signal Processing, vol. 41, pp. 2850-2862, Sept. 1993.

[8] W. Rudin, Principles of Mathematical Analysis. New York: McGraw-Hill, 1976.

[9] A. W. van der Vaart, Asymptotic Statistics. Cambrige, UK: Cambridge University Press, 1998.

[10] C. R. Rao, Linear Statistical Inference and Its Applications. New York: Wiley, 2002.

[11] A. Zeira and A. Nehorai, "Frequency domain Cramer-Rao bound for Gaussian processes," IEEE Trans. Signal Processing, vol. 38, pp. 1063-1066, June 1990.

[12] H. L. V. Trees, Detection, Estimation and Modulation Theory, vol. 1. New York: Wiley, 1968.

[13] F. Athley, "Threshold region performance of Maximum Likelihood direction of arrival estimators," IEEE Trans. Signal Processing, vol. 53, pp. 1359-1373, Apr. 2005.

[14] H. Lilliefors, "On the Kolmogorov-Smirnov test for normality with mean and variance unknown," Journal of the American Statistical Association, vol. 62, pp. 399-402, 1967.

[15] W. J. Conover, Practical Nonparametric Statistics. New York: Wiley, 1980. 